
\title{
Freestanding few-layer sheets of a dual topological insulator
}

\author{
Mai Lê Anh ${ }^{1}$, Pavel Potapov ${ }^{2}$, Axel Lubk ${ }^{2,3}$, Thomas Doert $\mathbb{D}^{1}$ and Michael Ruck $\mathbb{D}^{1,3,4 凶}$
}

The emergence of topological insulators (TIs) raised high expectations for their application in quantum computers and spintronics. Being bulk semiconductors, their nontrivial topology at the electronic bandgap enables dissipation-free charge and spin transport in protected metallic surface states. For application, crystalline thin films are requested in sufficient quantity. A suitable approach is the liquid phase exfoliation (LPE) of $\mathrm{TI}$ crystals that have layered structures. $\mathrm{Bi}_{2}$ Tel is a weak $3 \mathrm{D} \mathrm{TI}$, which leads to protected edge states at the side facets of a crystal, as well as a topological crystalline insulator, which is responsible for protected states at the top and bottom faces. We developed an effective, scalable protocol for LPE of freestanding nanoflakes from $\mathrm{Bi}_{2} \mathrm{Tel}_{\mathrm{crystals}}$. By heat treatment and sonication in isopropyl alcohol and poly(vinylpyrrolidone), crystalline $\mathrm{Bi}_{2}$ Tel sheets with a thickness of $\sim 50 \mathrm{~nm}$ were obtained and can therefore be considered for further processing toward microelectronic applications.

npj 2D Materials and Applications (2021)5:22 ; https://doi.org/10.1038/s41699-021-00203-6

\section{INTRODUCTION}

Topological insulators (TIs) are a relatively new class of quantum materials, which are currently heavily investigated for their intriguing fundamental properties, as well as possible applications in future electronic and spintronic devices. Highly mobile electrons that are protected against backscattering by timeinversion symmetry and topology exist on the surface of $\mathrm{Tls}^{1-3}$. Their spin is locked orthogonal to their propagation direction, i.e., attaching a spin current to electrons traveling into a particular direction. These two properties make Tls highly desirable materials for quantum computing and spintronics ${ }^{4,5}$ by virtue of the topology and symmetry of their band structure ${ }^{1,6}$. A TI must be a bulk semiconductor with nontrivial $\mathbb{Z}_{2}$ invariants (one in $2 \mathrm{D}$, four in 3D TIs), describing how often the surface bands cross the bandgap (in various directions). This so-called nontrivial topology at the bandgap can be achieved either by hybridization or by strong spin-orbit coupling and can persist at room temperature ${ }^{7,8}$. According to the $\mathbb{Z}_{2}$ classification and dimensionality, Tls may be classified in to 2D Tls (a quantum spin Hall state) and 3D strong and weak TIs (WTI). The latter exhibit topologically nontrivial surface states only at particular surfaces (indicated by the invariants) and may be obtained from stacking 2D TIs along the out-of-plane direction.

Applications in micro- or nanoelectronics require thin TI films of high quality, as well as a reasonable processing and scalability. Common techniques include chemical and physical vapor deposition $^{9,10}$ and molecular beam epitaxy ${ }^{11,12}$. The latter allows very good control, but is complicated and expensive, and thus can hardly be used in industrial processes. It appears more attractive to use top-down methods, in which $\mathrm{TI}$ films are exfoliated from high quality crystals. Some review articles summarize the attempts to exfoliate layered TI materials with a simple micromechanical cleavage technique using adhesive tape ${ }^{13,14}$. Even atomic force microscopy was used for the exfoliation of TI materials ${ }^{15}$. However, both procedures are either hard to control or extremely timeconsuming, and thus inefficient for production on larger scale. Instead of manual cleavage, liquid phase exfoliation (LPE) by sonication ${ }^{16,17}$, can be implemented to prepare thin sheets of
$\mathrm{TIs}^{18}$. LPE has the great advantage of being scalable and of producing freestanding flakes in a dispersion, which can then be processes with common techniques, such as spin-coating or printing.

This work focuses on the exfoliation of $\mathrm{Bi}_{2} \mathrm{Tel}$, which is an exceptional topological quantum material ${ }^{19,20}$ exhibiting coexisting topological surface states. The crystal structure of $\mathrm{Bi}_{2} \mathrm{Tel}$ is a stack of uncharged three atoms thick [TeBil] layers and puckered bismuth layers with the periodic sequence $[\mathrm{TeBil}]-\left[\mathrm{Bi}_{2}\right]-[\mathrm{IBiTe}]$ (Fig. 1) ${ }^{21,22}$. Each of these sandwich-like layer packages is a 2D TI with metallic edge states protected by time-inversion symmetry and a calculated bulk bandgap of $80 \mathrm{meV}$ (ref. ${ }^{23}$ ). Stackings of these $2 \mathrm{D} \mathrm{TI}$ layers result in a $3 \mathrm{D} \mathrm{WTI}$ (weakly interacting $\mathrm{TI}$ ). $\mathrm{Bi}_{2} \mathrm{Tel}$ is also a topological crystalline insulators (TCls), for which the protecting symmetry are mirror planes ${ }^{24}$. This creates separate topologically protected surface states on the top and bottom surfaces of the crystal $^{19}$. Here, we present a scalable chemical exfoliation strategy that yields freestanding few-layer sheets of the dual $\mathrm{Tl} \mathrm{Bi}_{2}$ Tel with high structural definition and crystallinity. Dispersions of these flakes allow the large-scale production of thin films and diverse further research on such films, which could pave the way to applications in quantum computing or spintronics.

\section{RESULTS}

\section{Chemical treatment of $\mathbf{B i}_{2}$ Tel crystals}

In a typical LPE, the layered material is immersed into a stabilized liquid containing a suitable solvent and surfactant ${ }^{16,17}$. Thereby, it is anticipated that the solvent induces swelling by an inclusion of suitable solvent molecules in the interlayer spaces. In addition, the surfactant can adsorb through non-covalent interactions at the layer interface of the material, further sensitizing the material to shear forces and exfoliation. Common solvents that are used for LPE are, for example, N-methyl-2-pyrrolidone (NMP), dimethyl sulfoxide (DMSO), and isopropyl alcohol (IPA) ${ }^{16}$. In its simplest and ideal way, the sole solvent already promotes swelling.

For this reason, carefully selected $\mathrm{Bi}_{2}$ Tel crystals were immersed in each of these solvents and heated to a certain temperature,

\footnotetext{
${ }^{1}$ Faculty of Chemistry and Food Chemistry, Technische Universität Dresden, Dresden, Germany. ${ }^{2}$ Leibniz Institute for Solid State and Materials Research, Dresden, Germany. ${ }^{3}$ Würzburg-Dresden Cluster of Excellence ct.qmat, c/o Technische Universität Dresden, Dresden, Germany. ${ }^{4}$ Max Planck Institute for Chemical Physics of Solids, Dresden, Germany.

email: michael.ruck@tu-dresden.de
} 


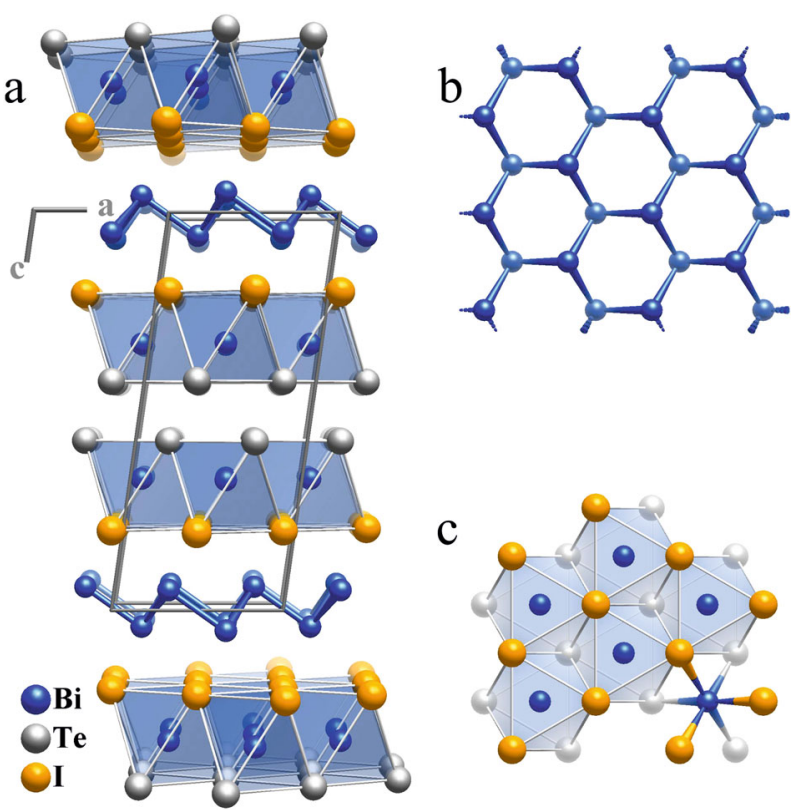

Fig. 1 Crystal structure of $\mathbf{B i}_{2}$ Tel. a Projection along the $b$-axis. $\mathbf{b}$ Top view on a $\left[\mathrm{Bi}_{2}\right]$ layer and $\mathbf{c}$ a [TeBil] layer.

\begin{tabular}{|lccl}
\hline $\begin{array}{l}\text { Table 1. } \\
\mathrm{Bi}_{2} \text { Tel. }\end{array}$ & List of solvents and reaction conditions tested to exfoliate \\
\hline Solvent & $\vartheta\left({ }^{\circ} \mathrm{C}\right)$ & $t(\mathrm{~h})$ & SEM/EDX results \\
\hline NMP & $80^{\text {a }}$ & 24 & Shifting of layer stacks \\
& 100 & 24 & Shifting of layer stacks \\
& 100 & 48 & Shifting and splitting \\
& 140 & 24 & Decomposition \\
DMSO & $60^{\text {a }}$ & 24 & Shifting of layer stacks \\
& 100 & 48 & Shifting and splitting \\
& 100 & 72 & No significant difference to the above \\
& $60^{\text {a }}$ & 24 & Shifting of layer stacks \\
IPA & 60 & 48 & Shifting and splitting \\
& 80 & 48 & No significant difference to the above \\
& 80 & 72 & No significant difference to the above \\
\hline \multicolumn{6}{c}{} \\
\hline aSEM image is depicted in Fig. 2 as an example of the morphology of the \\
Bi ${ }_{2}$ Tel crystals after the treatment.
\end{tabular}

depending on the individual boiling points. In order to monitor their effect on the exfoliation extent, the reaction mixtures were not stirred and no shear force or sonication was applied. Besides temperature, the reaction time was varied. The different reaction parameters are listed in Table 1. Scanning electron microscopy (SEM) analyses revealed that in all three solvents, the crystals fanned out and separated into multilayer stacks (Fig. 2). Powder Xray diffraction (PXRD) patterns of the swollen crystals matched well with the simulated diffraction pattern of $\mathrm{Bi}_{2} \mathrm{Tel}$ (Fig. 3), when taking into consideration the slight variation of the reflection intensities due to preferred orientation of the crystallites. Energydispersive X-ray (EDX) analyses showed the composition Bi:Te:I = 47(3):26(2):27(1) at.\%, which corresponds to the calculated ratio of 50:25:25 at.\% within the achievable accuracy. These preliminary experimental results on $\mathrm{Bi}_{2} \mathrm{Tel}$ proved that the crystals can be swollen although not to a large extend. Since similar effects had been obtained in all three solvents, we then focused on IPA, the least toxic and most sustainable chemical of them. Moreover, IPA can much easier be purified than the other solvents.

As mentioned, the use of surfactants is often absolutely necessary, not only to stabilize the resulting product, but also to interact at the interface between solvent and flake, thereby reducing the exfoliation energy and thus improving the swelling and splitting behavior. In the case of $\mathrm{Bi}_{2} \mathrm{Tel}$, highly polar solvents and surfactants should preferably bond to the surface of the also polar $\left[\mathrm{Te}^{2-} \mathrm{Bi}^{3+} \mathrm{I}^{-}\right]$layer (calculated electric polarization $P=$ $\left.0.069 \mathrm{C} \mathrm{m}^{-2}\right)^{25}$, and thus induce splitting at the desired structural gap between two [TeBil] layers (but not between [TeBil] and $\left[\mathrm{Bi}_{2}\right]$ ).

On that account, $\mathrm{Bi}_{2}$ Tel crystals were heated with PVP in IPA at $60^{\circ} \mathrm{C}$ for $48 \mathrm{~h}$. Indeed, the application of the surfactant PVP was found to be beneficial for the separation of the layered material. SEM images showed considerably expanded crystals, often with an accordion-like morphology (Fig. 4). Also, smaller flakes were observed which most probably set off during the spontaneous expansion. These flakes are obviously rather thin as they are transparent in the electron beam of the SEM (acceleration voltage $2 \mathrm{kV}$ ). Local EDX measurements on large and small flakes indicated a ratio of Bi:Te:I $=45(2): 29(2): 26(2)$ at.\%, which confirmed the chemical composition $\mathrm{Bi}_{2} \mathrm{Tel}$. We interpret these results as a proof for the chemical and mechanical robustness of the layered material.

\section{Sonochemical treatment}

Since the swollen crystals seemed to be shear labile and prone to exfoliation, they were exposed to ultra-sonication for $3 \mathrm{~h}$. Macroscopically, i.e., as visible to the naked eye, a gray dispersion had formed after $10 \mathrm{~min}$ of sonication. Subsequently, the dispersion was separated into particle size-dependent fractions by ultracentrifugation. Thin flakes with an estimated thickness of $\sim 50 \mathrm{~nm}$, i.e., 30 [TeBil] $-\left[\mathrm{Bi}_{2}\right]-[\mathrm{IBiTe}]$ layer packages of $1.58 \mathrm{~nm}$ (ref. ${ }^{23}$ ), were characterized via transmission electron microscopy (TEM; Fig. 5). Their lateral dimensions ranged from 0.5 to $5 \mu \mathrm{m}$, deduced from at least 25 individually deposited particles. Selected area electron diffraction patterns are in perfect agreement with simulated electron diffraction patterns of $\mathrm{Bi}_{2} \mathrm{Tel}$ (Fig. 6) concerning the geometry of the bulk unit cell. Moreover, HRTEM images of two distinct zones show atomic columns in agreement with the bulk structure, emphasizing the high crystallinity of the final flakes. EDX analyses of selected few-layer sheets revealed the chemical composition of Bi:Te:I=51(3):22.4(1):26(2) at.\%. All in all, $\mathrm{Bi}_{2} \mathrm{Tel}$ had also survived the sonication treatment undamaged. Composition and even the crystal structures of the isolated few-layer sheets were the same as in the initial $\mathrm{Bi}_{2}$ Tel crystals. For that reason, the $\mathrm{TCl}$ property and the $\mathrm{WTI}$ properties of the dual topological quantum material are expected to be also preserved.

\section{DISCUSSION}

The scalable protocol developed in this work concentrates on the LPE of the dual $\mathrm{TI} \mathrm{Bi}_{2} \mathrm{Tel}$ and comprises two steps. Firstly, the layered bulk material is immersed in a stabilizing liquid composed of an appropriate solvent (IPA) and a surfactant (PVP), and heated for a defined period of time. Thereby, swelling, shearing, and partial separation are induced. Secondly, the reaction mixture is sonicated to promote exfoliation. We succeeded in creating freestanding, i.e., not substrate supported, few-layer sheets of $\mathrm{Bi}_{2}$ Tel with the same high chemical and structural definition as in the initial crystals. Further physical characterization, especially by scanning tunneling microscopy, has to be done to reveal the surface termination and the transport properties of these few-layer sheets. Quantum theoretical calculations should help to evaluate the influence of terminations on the properties depending on the number of $\mathrm{Tl}$ layer packages. Dispersions of exfoliated dual $\mathrm{TI}_{\mathrm{Bi}} \mathrm{Bel}^{\mathrm{T}}$ sheets now allow for next steps toward the envisioned application 

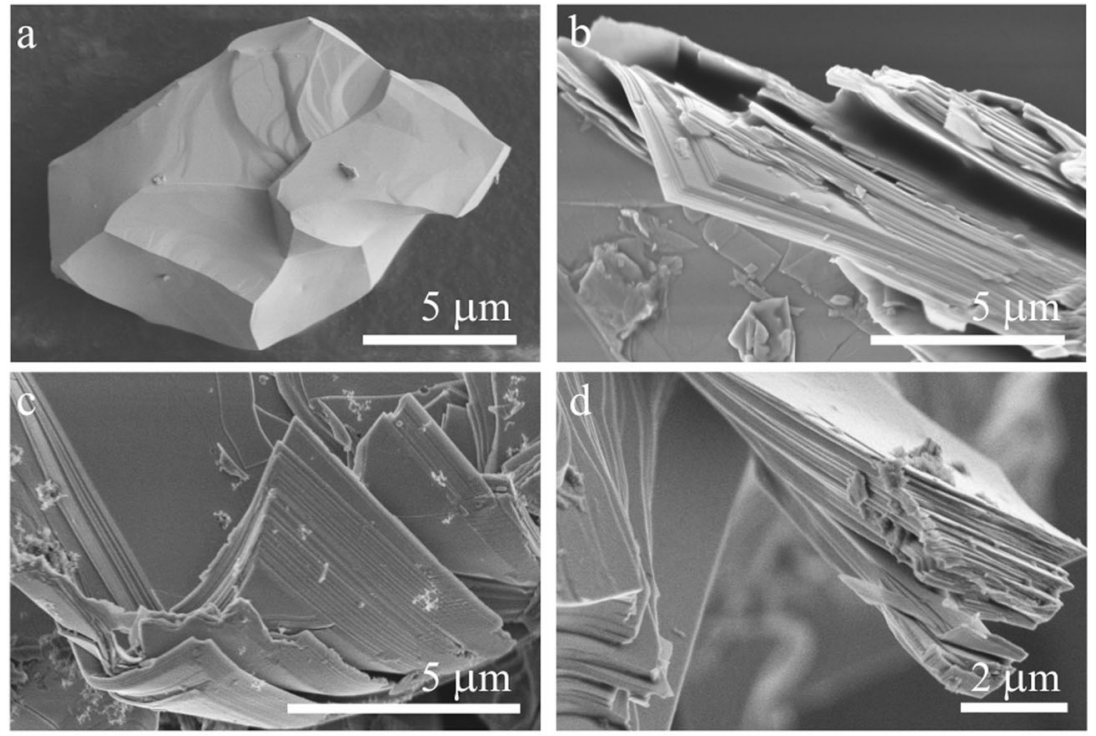

Fig. 2 SEM images demonstrating the results of the chemical treatment of $\mathbf{B i}_{2}$ Tel crystals. $\mathbf{a}$ Untreated $\mathrm{Bi}_{2} T e l$ crystals. $\mathbf{b}$ Bi ${ }_{2} T e l$ crystals after the heat treatment in DMSO, c NMP, or d IPA.
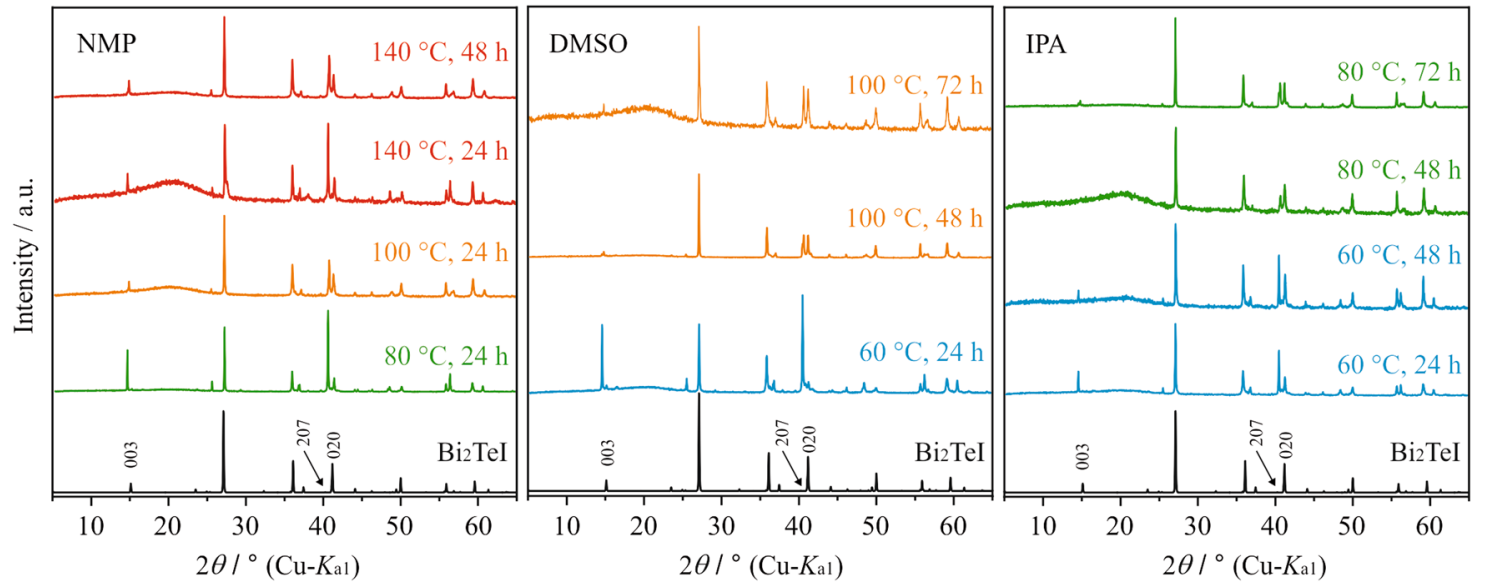

Fig. 3 PXRD patterns demonstrating the results of the chemical treatment of $\mathbf{B i}_{2} \mathbf{T e l}$. Products obtained from reactions at different temperatures and with different reaction times in NMP, DMSO, or IPA are compared to the simulated diffraction pattern of $\mathrm{Bi}_{2}$ Tel.

in nanoelectronics, such as the study of quantum coherence in deposited films, the combination with superconducting particles or films for the generation of Majorana fermions, or studies on their behavior under the influence of magnetic or electric fields, or in contact with various materials occurring in devices.

\section{METHODS}

\section{Synthesis of $\mathrm{Bi}_{2}$ Tel crystals}

All starting materials and products were handled in an argon-filled glove box (MBraun; $p\left(\mathrm{O}_{2}\right) / p_{0}<1$ p.p.m., $p\left(\mathrm{H}_{2} \mathrm{O}\right) / p_{0}<1$ p.p.m.). To purify the materials, bismuth $(>99.9 \%$, Merck) and tellurium $(>99.999 \%$, ChemPur) were treated with hydrogen flow at 220 and $400^{\circ} \mathrm{C}$, while bismuth triiodide ( $>99.9 \%$, Sigma Aldrich) was sublimed at $200^{\circ} \mathrm{C}$ prior to the hightemperature reaction. To grow large $\mathrm{Bi}_{2}$ Tel crystals, a phase-pure powder had to be synthesize beforehand. Following a literature procedure ${ }^{21}$, a mixture of $\mathrm{Bi}, \mathrm{Te}$, and $\mathrm{Bil}_{3}$ with the molar ratio $\mathrm{Bi}: \mathrm{Te}: \mathrm{I}=2: 1: 1$ was ground in a ball mill for 25 min under argon atmosphere, sealed in a quartz glass ampoule, and heated with a rate of $2 \mathrm{~K} \mathrm{~min}^{-1}$ at $390^{\circ} \mathrm{C}$ for at least 5 days before quenching to room temperature. Once the phase purity was verified by PXRD, the crystal growth was initiated by annealing the powder with a rate of $2 \mathrm{~K} \mathrm{~min}^{-1}$ between 410 and $390^{\circ} \mathrm{C}$ in a two-zone furnace.

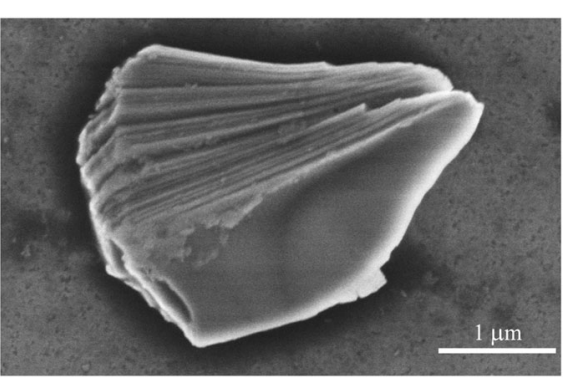

Fig. 4 Typical morphology of a swollen $\mathbf{B i}_{2}$ Tel crystal. SEM image of a swollen $\mathrm{Bi}_{2} \mathrm{Tel}$ crystal after the heat treatment with PVP in IPA, revealing expansion of the crystal and twisting of layer stacks.

After 1 week, the mixture was quenched to room temperature. All samples were handled under inert conditions.

\section{Liquid phase exfoliation of $\mathbf{B i}_{2} \mathbf{T e l}$}

The LPE experiments were executed in a specially designed glass tube for ultra-sonication application. To start the LPE reaction, $\mathrm{Bi}_{2} \mathrm{Tel}$ crystals with a 


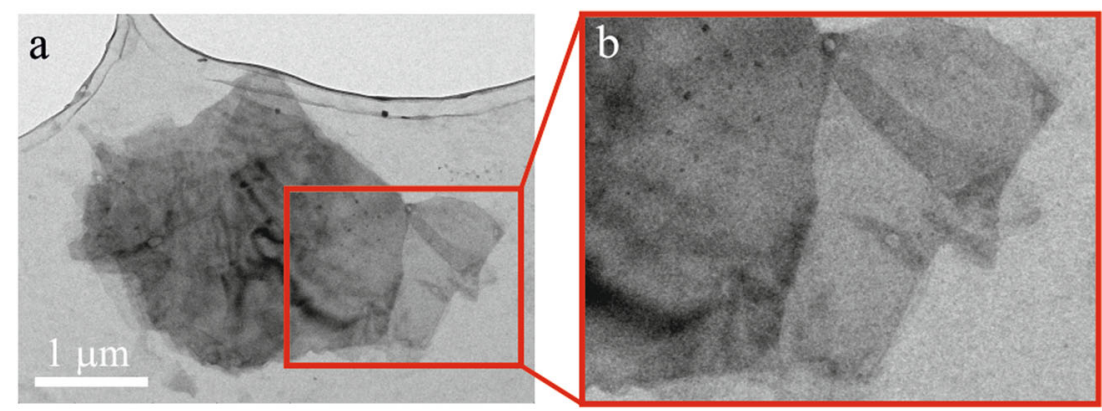

Fig. 5 TEM image of exfoliated few-layer sheets that appear almost transparent in the electron beam. a Flake obtained from swollen $\mathrm{Bi}_{2} \mathrm{Tel}$ crystals, which were heated in a mixture of PVP and IPA, and subsequently sonicated. b Detail in higher magnification.
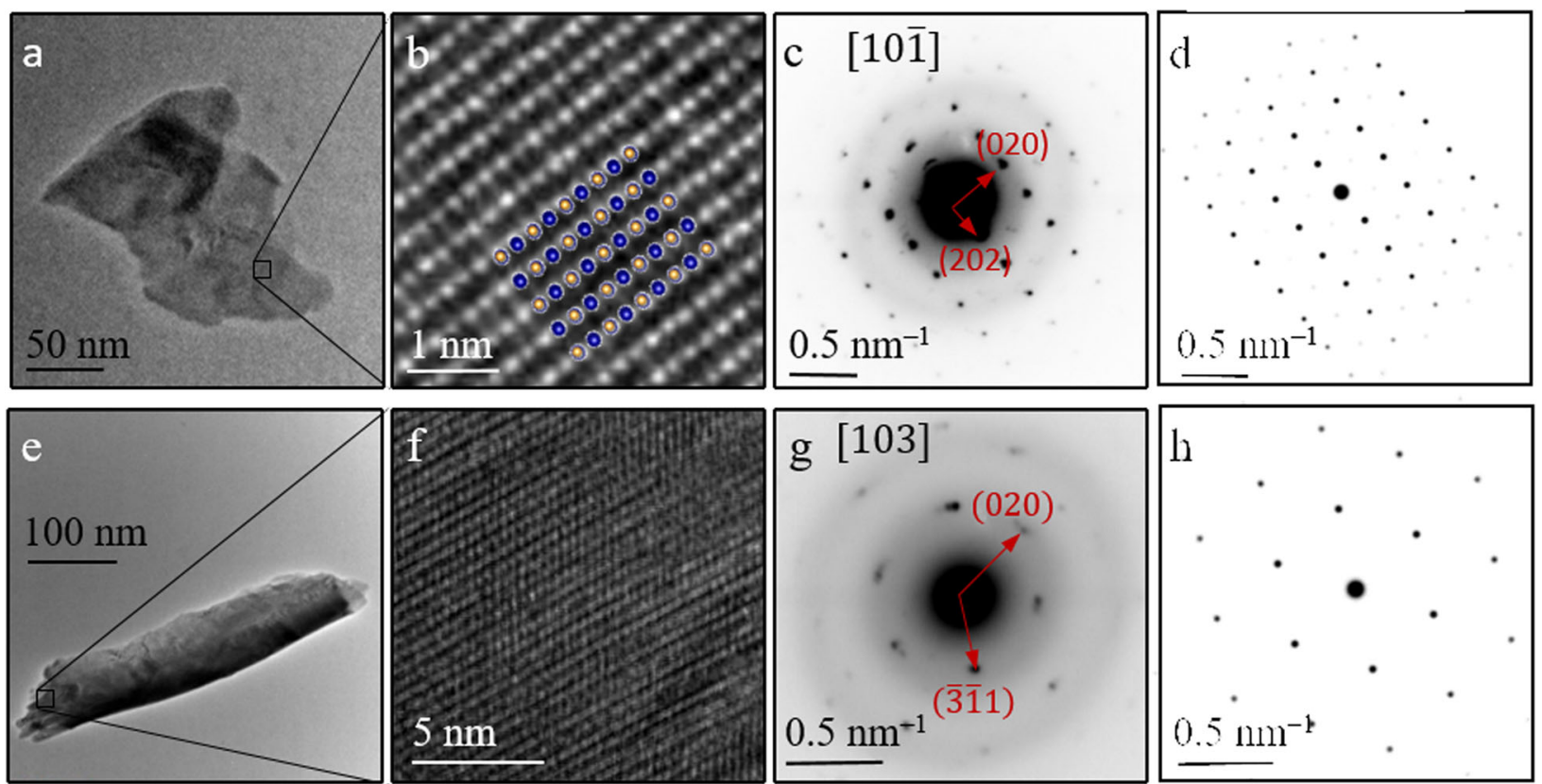

Fig. 6 Structural characterization of few-layer sheets of $\mathbf{B i}_{\mathbf{2}}$ Tel crystals. $\mathbf{a}$, e TEM images and $\mathbf{b}$, $\mathbf{f}$ HRTEM images demonstrate their high crystallinity. b HRTEM image with projected atomic columns in [10 $\overline{1}$ ] orientation, which are superimposed with the structure model, employing the color scheme from Fig. 1. Comparison of $\mathbf{c}, \mathbf{g}$ experimental and $\mathbf{d}$, $\mathbf{h}$ simulated electron diffraction patterns, exhibiting excellent agreement within the limits of the electron diffraction method. The simulation was based on the crystal structure of $\mathrm{Bi}_{2} \mathrm{Tel}$.

lateral dimension of at least $50 \mu \mathrm{m}$ were selected. A total of $0.016 \mathrm{mmol}$ $(10.8 \mathrm{mg})$ thereof and the same mass of PVP (Sigma Aldrich) were weighted into the glass tube. Correspondingly, the glass tube was carefully mounted onto the horn and flushed with argon gas. A volume of $8 \mathrm{ml}$ of NMP (Acros Organics), DMSO (AnalaR Normapur), or IPA (Sigma Aldrich) was then injected into the glass tube with an argon-flushed syringe in an argon countercurrent principle. A short sonomechanical treatment $(t=$ 20 s) was applied to homogenize the suspension and to fully cover the particle surfaces with the reaction media. The reaction mixture was subsequently heated to maximally $60^{\circ} \mathrm{C}$ for at least $24 \mathrm{~h}$ and treated with ultrasound (Bandelin Sonopuls, sonication parameters: $t_{\max }=3 \mathrm{~h}, E_{\max }=$ $154 \mathrm{~kJ})$. After cooling down, a part of the final product was washed repeatedly with absolute ethanol in an argon-filled glove box and size selected via ultracentrifugation (Beckman Coulter, centrifugation parameters: $t_{\max }=50 \mathrm{~min}, f_{\max }=20.000 \mathrm{~min}^{-1}$ ), while the other part was left untreated for comparative investigations. All products were stored under inert argon.

\section{Powder X-ray diffraction}

The PXRD data ( $\mathrm{CuKa}_{1}, \lambda=1.540562 \AA, T=296(1) \mathrm{K}$ ) were collected using an X'Pert Pro diffractometer (PANalytical, Bragg-Brentano geometry, Ge (220) hybrid monochromator, fixed divergence slits, PIXcel detector). The samples were fixed on single-crystal silicon sample holders. The Powder
Diffraction File database and the Inorganic Crystal Structure database were used for phase identification.

\section{Scanning electron microscopy and energy-dispersive X-ray spectroscopy}

SEM was performed using an electron microscope SU8020 from Hitachi equipped with multi detector system for secondary and low-energy backscattered electrons, while an Oxford Silicon Drift Detector X-Max ${ }^{\mathrm{N}}$ was used for the semiquantitative EDX spectroscopy. Suspensions washed with ethanol were dropped on a silicon wafer, which were then settled on an aluminum sample holder with an adhesive carbon pad. The EDX data were evaluated with the AZtec software package ${ }^{26}$. Variations in the EDX data were mainly caused by slightly tilted crystal surfaces, as it was difficult to align the flakes exactly parallel to the electron beam.

\section{Transmission electron microscopy}

TEM imaging was either performed in a JEOL JEM-1400 microscope at $120 \mathrm{kV}$ accelerating voltage or in a FEl Titan ${ }^{3}$ 80-300 (ThermoFisher Company) microscope at $300 \mathrm{kV}$ acceleration voltage. The instrument was equipped with an image aberration corrector allowing for a spatial resolution down to $0.7 \AA$ in the bright field mode. The diffraction patterns were recorded using the three-lens condenser system to form parallel 
illuminated probes of $\sim 400 \mathrm{~nm}$ in diameter. These relatively large probes allowed to reduce significantly the beam-induced damage. To index the diffraction patterns, we employed the SingleCrystal software package (CrystalMaker Software LtD, UK) allowing for simulating electron diffraction patterns. For conventional TEM measurements, the samples were prepared by diluting dispersions and drop-casting onto a copper grid with subsequent evaporation of the solvent.

\section{DATA AVAILABILITY}

The data that support the findings of this study are available from the corresponding author on reasonable request.

Received: 28 September 2020; Accepted: 19 January 2021; Published online: 18 February 2021

\section{REFERENCES}

1. Kane, C. L. \& Mele, E. J. $Z_{2}$ Topological order and the quantum spin hall effect. Phys. Rev. Lett. 95, 146802 (2005).

2. König, M. et al. Quantum spin hall insulator state in HgTe quantum wells. Science 318, 766-770 (2007).

3. von Klitzing, K. Developments in the quantum Hall effect. Philos. Trans. R. Soc Lond. 363, 2203-2219 (2005).

4. He, M., Sun, H. \& He, Q. L. Topological insulator: spintronics and quantum computations. Front. Phys. 14, 43401 (2019).

5. Aspuru-Guzik, A. \& Walther, P. Photonic quantum simulators. Nat. Phys. 8 285-291 (2012)

6. Qi, X.-L. \& Zhang, S.-C. Topological insulators and superconductors. Rev. Mod. Phys. 83, 1057-1110 (2011).

7. Hasan, M. Z. \& Kane, C. L. Colloquium: topological insulators. Rev. Mod. Phys. 82 3045-3067 (2010).

8. Wang, $\mathrm{H}$. et al. Ferromagnetic dual topological insulator in a two-dimensional honeycomb lattice. Mater. Horiz. 7, 2431-2438 (2020).

9. Kong, D. et al. Topological insulator nanowires and nanoribbons. Nano Lett. 10, 2245-2250 (2010).

10. Liu, M., Liu, F. Y., Man, B. Y., Bi, D. \& Xu, X. Y. Multi-layered nanostructure $\mathrm{Bi}_{2} \mathrm{Se}_{3}$ grown by chemical vapor deposition in selenium-rich atmosphere. Appl. Surf. Sci. 317, 257-261 (2014).

11. Chen, X., Ma, X.-C., He, K., Jia, J.-F. \& Xue, Q.-K. Molecular beam epitaxial growth of topological insulators. Adv. Mater. 23, 1162-1165 (2011).

12. Ginley, T. P., Wang, Y. \& Law, S. Topological insulator film growth by molecula beam epitaxy: a review. Crystals 6, 154 (2016).

13. Gupta, B. K. et al. Unexplored photoluminescence from bulk and mechanically exfoliated few layers of $\mathrm{Bi}_{2} \mathrm{Te}_{3}$. Sci. Rep. 8, 9205 (2018).

14. $\mathrm{Yu}, \mathrm{W}$. et al. Magneto-infrared study of topological insulator $\mathrm{Bi}_{2} \mathrm{Se}_{3}$. Preprint at https://arxiv.org/abs/1508.04363 (2015).

15. Hong, S. S. et al. Ultrathin topological insulator $\mathrm{Bi}_{2} \mathrm{Se}_{3}$ nanoribbons exfoliated by atomic force microscopy. Nano Lett. 10, 3118-3122 (2010)

16. Backes, C. et al. Guidelines for exfoliation, characterization and processing of layered materials produced by liquid exfoliation. Chem. Mater. 29, 243-255 (2017).

17. Nicolosi, V., Chhowalla, M., Kanatzidis, M. G., Strano, M. S. \& Coleman, J. N. Liquid exfoliation of layered materials. Science 340, 1226419 (2013).

18. Fülöp, B. et al. Exfoliation of single layer BiTel flakes. 2D Mater. 5, 031013 (2018).

19. Avraham, N. et al. Visualizing coexisting surface states in the weak and crystalline topological insulator $\mathrm{Bi}_{2}$ Tel. Nat. Mater. 19, 610-616 (2020).

20. Pacchioni, G. One material, dual topology. Nat. Rev. Mater. 5, 332 (2020).

21. Zeugner, A. et al. Modular design with $2 \mathrm{D}$ topological-insulator building blocks: optimized synthesis and crystal growth and crystal and electronic structures of $\mathrm{Bi}_{x} \mathrm{Tel}(x=2,3)$. Chem. Mater. 29, 1321-1337 (2017).
22. Savilov, S. V., Khrustalev, V. N., Kuznetsov, A. N., Popovkin, B. A. \& Antipin, Yu. M. New subvalent bismuth telluroiodides incorporating $\mathrm{Bi}_{2}$ layers: the crystal and electronic structure of $\mathrm{Bi}_{2}$ Tel. Russ. Chem. Bull. 54, 87-92 (2005).

23. Tang, P. et al. Weak topological insulators induced by the interlayer coupling: a first-principles study of stacked $\mathrm{Bi}_{2}$ Tel. Phys. Rev. B 89, 041409 (2014).

24. Rusinov, I. P. et al. Mirror-symmetry protected non-TRIM surface state in the weak topological insulator $\mathrm{Bi}_{2}$ Tel. Sci. Rep. 6, 20734 (2016).

25. Kim, J., Rabe, K. M. \& Vanderbilt, D. Negative piezoelectric response of van der Waals layered bismuth tellurohalides. Phys. Rev. B 100, 104115 (2019).

26. AZtec, AZtec Version 2.2 SP1 (Oxford Instruments Technology Tools Ltd., 2013).

\section{ACKNOWLEDGEMENTS}

This work was supported by the Deutsche Forschungsgemeinschaft (DFG) within the Collaborative Research Center "Chemistry of Synthetic Two-Dimensional Materials" (SFB 1415, project-ID 417590517) and under Germany's Excellence Strategy through the Würzburg-Dresden Cluster of Excellence on "Complexity and Topology in Quantum Matter-ct.qmat" (EXC 2147, project-id 390858490).

\section{AUTHOR CONTRIBUTIONS}

M.L.A. and M.R. developed the ideas and the chemical procedure. M.L.A. performed the synthetic work and major parts of the characterization. Profound TEM measurements were carried out by P.P. and A.L. All authors contributed to the interpretation of the results and to the manuscript. All authors have approved the submitted version of this manuscript.

\section{FUNDING}

Open Access funding enabled and organized by Projekt DEAL.

\section{COMPETING INTERESTS}

The authors declare no competing interests.

\section{ADDITIONAL INFORMATION}

Correspondence and requests for materials should be addressed to M.R.

Reprints and permission information is available at http://www.nature.com/ reprints

Publisher's note Springer Nature remains neutral with regard to jurisdictional claims in published maps and institutional affiliations.

\begin{abstract}
Open Access This article is licensed under a Creative Common Attribution 4.0 International License, which permits use, sharing, adaptation, distribution and reproduction in any medium or format, as long as you give appropriate credit to the original author(s) and the source, provide a link to the Creative Commons license, and indicate if changes were made. The images or other third party material in this article are included in the article's Creative Commons license, unless indicated otherwise in a credit line to the material. If material is not included in the article's Creative Commons license and your intended use is not permitted by statutory regulation or exceeds the permitted use, you will need to obtain permission directly from the copyright holder. To view a copy of this license, visit http://creativecommons. org/licenses/by/4.0/.
\end{abstract}

(c) The Author(s) 2021 